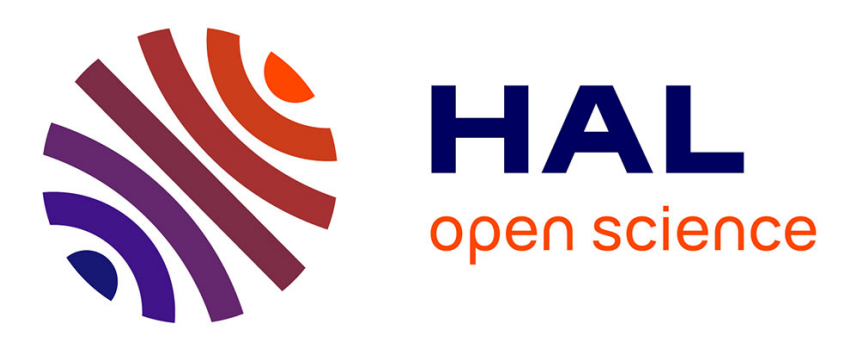

\title{
Les pratiques informationnelles individuelles et collectives des enseignants d'un collège rural. Une enquête exploratoire
}

Anita Messaoui

\section{- To cite this version:}

Anita Messaoui. Les pratiques informationnelles individuelles et collectives des enseignants d'un collège rural. Une enquête exploratoire. Spirale - Revue de Recherches en Éducation , 2016. hal01884940

\section{HAL Id: hal-01884940 \\ https://hal.science/hal-01884940}

Submitted on 1 Oct 2018

HAL is a multi-disciplinary open access archive for the deposit and dissemination of scientific research documents, whether they are published or not. The documents may come from teaching and research institutions in France or abroad, or from public or private research centers.
L'archive ouverte pluridisciplinaire HAL, est destinée au dépôt et à la diffusion de documents scientifiques de niveau recherche, publiés ou non, émanant des établissements d'enseignement et de recherche français ou étrangers, des laboratoires publics ou privés. 


\title{
LES PRATIQUES INFORMATIONNELLES INDIVIDUELLES ET COLLECTIVES DES ENSEIGNANTS D'UN COLLÈGE RURAL
}

\author{
UNE ENQUÊTE EXPLORATOIRE
}

\begin{abstract}
Résumé : L'approche documentaire du didactique (Gueudet et Trouche, 2010) a mis en évidence l'importance du travail sur les ressources pour l'enseignant. Cet aspect du métier d'enseignant est complètement bouleversé par les dispositifs numériques qui se sont développés depuis une dizaine d'années. La présente étude s'inscrit dans le cadre du projet de recherche ANR-ReVEA (Ressources vivantes pour l'enseignement et l'apprentissage). Elle est plus particulièrement centrée sur le travail documentaire des enseignants de mathématiques et d'anglais. L'article propose d'étudier les pratiques informationnelles des enseignants d'un collège rural en identifiant dans un premier temps les ressources mobilisées par les enseignants pour préparer et faire la classe. L'enquête apporte ensuite des éléments sur la dimension collective du travail documentaire des enseignants de ce collège, dans les équipes disciplinaires, mais aussi dans un contexte de travail interdisciplinaire. Enfin, ces premiers éléments sont mis en perspective à la lueur des livrables déjà publiés par les acteurs de de la recherche ReVEA.

Mots-clefs : pratiques informationnelles, approche documentaire du didactique, ressources éducative, travail collectif/recherche en ligne, enseignement des mathématiques, enseignement de l'anglais.
\end{abstract}

\section{INTRODUCTION}

La présente étude est la première pierre d'un travail de thèse débuté en octobre 2015. Il s'agit d'une enquête exploratoire destinée à préciser nos questions de recherche. Ce travail s'inscrit dans le projet de recherche ANR-ReVEA ${ }^{1}$ (Ressources vivantes pour l'enseignement et l'apprentissage). L'un des objectifs de cette recherche est d'accéder à une meilleure compréhension des évolutions de la profession dans un contexte de reconfiguration des ressources utilisées avec, notamment, la généralisation de l'usage du numérique. Au sein de ce projet national, nous nous intéressons plus particulièrement à la manière dont les enseignants recherchent, sélectionnent et agrègent de nouvelles ressources dans un même établissement (tâche 3). L'ensemble des équipes ReVEA travaillent avec des enseignants du secondaire de quatre disciplines : mathématiques, anglais, sciences et techniques industrielles (STI), et sciences physiques. La mise en œuvre de la réforme du collège de septembre 2016 prévoit un renouvellement des programmes sur tous les niveaux du collège. Elle introduit de nouveaux dispositifs pédago-

${ }^{1}$ https://www.anr-revea.fr/ 
giques $^{2}$ et propose un référentiel pour l'éducation aux médias et à l'information (EMI). Cette EMI, enseignement transversal, est de la responsabilité de tous les enseignants. Une partie du référentiel EMI s'appuie sur les sciences de l'information et de la communication (SIC) dont les professeurs ne sont pas familiers. Cependant, de par leurs pratiques, ils mettent en œuvre dans leur activité professionnelle des compétences relevant de l'EMI. Demander aux élèves de contextualiser un document, de faire une recherche documentaire ou de sélectionner une information dans un document pour répondre à une question, autant d'activités courantes qui relèvent toutes du champ de l'EMI. Dans notre étude doctorale, nous questionnons les compétences spécifiques aux traitements de l'information à l'œuvre dans le travail documentaire des enseignants, et analysons comment cette $e x$ pertise documentaire peut influer sur la mise en œuvre de l'EMI par les acteurs de l'enseignement. Nous émettons l'hypothèse que le travail documentaire des enseignants est intimement lié au développement de leurs connaissances professionnelles. Afin de mener à bien ce projet doctoral, nous avons commencé par mener une enquête par questionnaire sur l'ensemble de la population enseignante d'un collège rural. Cette enquête a permis de dresser une cartographie des pratiques informationnelles de la population. Dans un second temps, nous avons invité les enseignants à discuter les résultats de ce questionnaire lors d'une réunion de présentation.

Après avoir expliqué le cadre théorique retenu et la méthodologie mise en œuvre, nous définissons les caractéristiques de la population étudiée. Nous présentons ensuite les résultats de l'enquête selon trois axes. Tout d'abord, nous dressons une typologie des ressources utilisées pour préparer et faire la classe : quelles sont ces ressources et remarque-t-on des spécificités disciplinaires ? En ce qui concerne les deux derniers axes, nous analysons la dimension collective du travail documentaire sur le plan disciplinaire puis interdisciplinaire.

\section{CADRE THÉORIQUE ET PROBLÉMATIQUE}

Pour commencer, nous souhaitons clarifier les concepts qui seront utilisés par la suite et en particulier le terme de ressource. Étymologiquement, ressource vient «du lat. resurgere, de re, et surgere, se lever, surgir. La ressource est proprement une seconde ou dernière source, une chose qui relève, un moyen qui fait sortir d'embarras » ${ }^{3}$. Il s'agit donc d'un élément tiers, extérieur qui va contribuer à améliorer une situation. Etudier les pratiques informationnelles des enseignants, c'est observer et analyser comment ils travaillent avec les ressources dans leur activité d'enseignement.

Pour mener cette étude, nous prenons appui sur l'approche documentaire du didactique (Gueudet \& Trouche, 2010). En partant de l'approche instrumentale (Rabardel, 1995), Gueudet \& Trouche proposent un cadre d'analyse du travail documentaire des enseignants. Nous utilisons donc le terme ressource dans l'acceptation qui lui est donnée par ces auteurs : « un artefact : un produit de l'activité humaine, élaboré pour s'inscrire dans une activité finalisée. » (Gueudet \& Trouche 2010 : 58) Cette définition relativement large met l'accent sur l'usage, plutôt que sur une typologie qui serait nécessairement réductrice. Dans cette approche, le travail documentaire est un "processus articulant étroitement concep-

\footnotetext{
${ }^{2}$ Les enseignements pratiques interdisciplinaires (EPI) et l'accompagnement personnalisé (AP)

${ }^{3}$ Dictionnaire Littré : http://www.littre.org/definition/ressource
} 
tion et mise en auvre $»^{4}$ des ressources utilisées par l'enseignant. Le travail documentaire englobe la recherche, l'agrégation et la création de ressources. Le processus spécifique consistant à adapter une ressource au besoin de l'enseignant est appelé genèse documentaire. Il en résulte un document qui est constitué d'un ensemble de ressources recomposées et des schèmes d'utilisation (Vergnaud, 1996) que l'enseignant a développés. Ainsi, un usage potentiel est associé au document élaboré. L'ensemble des ressources disponibles et des documents produits par l'enseignant constitue son système de ressources.

La réalisation du travail documentaire met en jeu des pratiques informationnelles. Chaudiron et Ihadjadene (2010:15) s'appuient sur les travaux de Jouët (1993) pour définir le terme de pratique qu'ils distinguent de celui d'usage :

«La notion de pratique est plus élaborée que celle d'usage, car elle prend en compte l'utilisation des techniques, mais également les comportements, les attitudes, les représentations de ceux qui y ont recours (...) L'observation des pratiques, qu'elles soient individuelles ou collectives, nécessite alors d'adopter une approche de l'action envisagée comme un processus en tension entre les savoirs mobilisables, les compétences immédiates, les habitus, les arts de faire, les désirs d'agir. »

La pratique se place donc du côté du faire et de l'agir dans une perspective concrète. Tous les éléments mobilisés dans la pratique, les savoirs, les techniques, les manières d'être, visent une action concrète efficace. Ce terme convient donc bien pour décrire le travail documentaire des enseignants, la finalité étant la production de documents efficients pour enseigner. Gardies et al. (2010) insistent également sur la dimension sociale incluse dans le terme de «pratique » : les pratiques professionnelles sont partagées par les acteurs d'une communauté et elles s'enrichissent mutuellement des apports des uns et des autres.

Le travail documentaire est ici analysé selon une perspective info-communicationnelle (Chaudiron \& Ihadjadene, 2010). Au-delà des questions de la recherche et de l'accès à l'information, nous considérons également les dimensions éthiques, économiques et identitaires de l'activité documentaire des enseignants. Il nous semble que la notion de document, telle que définie par Gueudet et Trouche (2008), c'est-à-dire la recomposition d'un ensemble de ressources associant des schèmes d'utilisation s'inscrit bien dans cette perspective.

Nous avons longtemps hésité à utiliser le terme de «pratiques documentaires » puisque le centre de notre étude est le processus de production de document de travail par les enseignants. Nous y avons renoncé pour plusieurs raisons : d'une part, cette expression est souvent employée dans la littérature sans jamais être clairement définie (Alava, 1997 ; Pirolli, 2010). Lorsqu'une définition est donnée, elle est centrée sur le travail de gestion des documents (Maurel \& Chebbi 2012; Wojciechowska, 2012) ou bien circonscrite aux comportements de recherche documentaire (Bouzon et al., 1996 ; Boubée, 2007). D'autre part, limiter notre étude à la sphère documentaire nous semblait trop réducteur. Si l'aboutissement du travail documentaire est bien un document, la genèse qui y mène mobilise tout le champ des pratiques informationnelles. La dimension informationnelle renvoie tout à la fois à la connaissance des sources, aux interactions interpersonnelles, aux processus de recherche, au traitement de l'information sélectionnée ainsi qu'aux documents produits par les enseignants. Nous utilisons donc le terme de pratiques in-

\footnotetext{
${ }^{4}$ Ibid. p. 58
} 
formationnelles dans l'acceptation que lui donnent Chaudiron et Ihadjadene $(2010: 14)$ :

« [ce terme] désigne la manière dont un ensemble de dispositifs, de sources formelles ou non, d'outils, de compétences cognitives sont effectivement mobilisés, par un individu ou un groupe d'individus, dans les différentes situations de production, de recherche, d'organisation, de traitement, d'usage, de partage et de communication de l'information. »

Le cadre théorique convoqué ici fait appel à la fois aux sciences de l'éducation et aux sciences de l'information et de la communication. Nous nous plaçons donc dans une perspective double. La première interroge la manière dont les enseignants recherchent et utilisent des informations, leurs pratiques informationnelles. La seconde examine comment ces pratiques informationnelles sont liées aux contenus d'enseignement mis en œuvre par les acteurs de terrain. Nous tenterons de répondre aux questions suivantes : quels types de documents sont utilisés par les enseignants pour faire la classe ? Comment les enseignants cherchent-ils puis sélectionnent-ils de nouvelles ressources ? Comment stockent-ils et organisent-ils leurs ressources ? Quels liens existe-t-il entre le travail documentaire de l'enseignant et son système de connaissances ? Quelle est la part collective dans le travail documentaire et de quel(s) collectif(s) s'agit-il ?

Notre travail de thèse fait suite à un mémoire de master sur les usages du numérique des enseignants (Messaoui, 2014). L'usage exclusif des manuels y était remis en cause par plusieurs acteurs. Notre première hypothèse porte donc sur la diversification des ressources utilisées par les enseignants et notamment la présence renforcée de ressources numériques (H1). Notre seconde hypothèse est corollaire de la première. Cette diversification des sources s'accompagne d'une intensification des pratiques d'Internet dans le cadre professionnel (H2). Une étude de Dioni (2008) pointait encore des blocages pour une partie des enseignants. Nous pensons que ces blocages sont dépassés pour une majorité des enseignants du secondaire. La troisième hypothèse est d'ordre qualitatif : les enseignants les moins expérimentés sont ceux qui recherchent le plus de ressources prêtes à l'emploi (H3). En début de carrière, lorsque les enseignants ont une documentation personnelle réduite, assurer les cours demande un important travail de préparation qui peut être avantageusement réduit en utilisant des ressources prêtes à l'emploi. Notre dernière hypothèse est en lien avec le projet de recherche ReVEA qui tente de discerner des affinités et des spécificités disciplinaires : la discipline d'enseignement a une influence sur l'intensité des échanges de ressources (H4).

\section{CONTEXTE ET MÉTHODOLOGIE GÉNÉRALE}

Le choix du terrain s'est porté sur le collège où nous avons exercé comme professeur-documentaliste. Ceci est un atout dans le sens où nous connaissons bien l'histoire de l'établissement et les jeux relationnels des acteurs de ce terrain. Cependant, les habitudes et les routines pourraient aussi biaiser notre regard. L'enquête par questionnaire est ainsi une manière de mettre à distance nos représentations du terrain et d'observer les différences entre ce que nous pensons connaître de la situation du collège et les pratiques déclarées par les enseignants.

Une méthodologie étagée

Le travail documentaire n'est pas directement accessible aux chercheurs et nécessite des méthodes d'investigations qualitatives fines sur un échantillon réduit d'acteurs. La méthodologie élaborée s'appuie sur la triangulation des données. Il s'agit de croiser différentes méthodes de recueil de données afin d'appréhender la 
complexité des situations du travail documentaire. Nous associons donc dans ce travail de thèse des méthodes basées sur des déclarations (questionnaire et entretiens), sur des observations filmées et sur des traces. L'investigation réflexive développée par Gueudet et Trouche (2010) occupe une place importante dans notre démarche. Il s'agit en effet d'observer les pratiques de travail intime des enseignants. L'observation des processus cognitifs s'effectue avec le concours des acteurs et suppose leur participation active : tenue d'un journal de bord, dispositif de captation vidéo du travail de l'enseignant sur son ordinateur, réalisation de cartes réflexives. Les éléments nécessairement subjectifs qui appartiennent aux « dires » des acteurs sont confrontés chaque fois que cela est possible à leurs actes ou aux traces de leurs productions. Cette méthodologie nécessite d'élaborer un contrat de confiance entre acteurs de terrain et chercheur (Sabra, 2016). Avant d'entamer un suivi individuel de quelques participants, nous avons souhaité avoir une vision d'ensemble de tous les acteurs du terrain.

La première étape de cette étude a donc été la diffusion d'un questionnaire (cf. annexe p. 75) à l'ensemble des professeurs de l'établissement en février 2015. Le questionnaire a été bâti à partir des hypothèses autour de quatre thématiques : les ressources pour la classe, le travail collectif, les projets pour la rentrée 2016 et l'histoire professionnelle. Ses objectifs sont multiples. Tout d'abord, il permet de dresser une cartographie des pratiques informationnelles à un instant « $t »$. Dans le contexte de la mise en place d'une réforme profonde du collège en septembre 2016, ces premiers résultats serviront de témoin pour apprécier des changements de pratiques potentiels. Le changement des programmes ${ }^{5}$, l'organisation par cycle et la mise en place de nouveaux dispositifs ${ }^{6}$ représentent un moment fondateur pour renouveler les ressources. La recherche ReVEA s'intéresse plus particulièrement aux enseignants de sciences physiques, STI, de mathématiques et d'anglais. Notre terrain d'étude est un collège situé en zone rurale à $60 \mathrm{~km}$ d'une métropole régionale. Afin de disposer d'un échantillon suffisant, nous proposons de suivre les enseignants des équipes de mathématiques et d'anglais. Pour l'enquête que nous présentons ici, nous avons fait le choix d'élargir l'échantillon au-delà de la population ciblée afin de développer une vision globale des différents champs disciplinaires. Nous espérons ainsi dégager des caractéristiques propres à chacune des disciplines cibles (anglais et mathématiques).

Sur le plan technique, le questionnaire a été dépouillé à l'aide du logiciel Modalisa. Chaque question a été analysée par tri à plat puis par tri croisé à partir de la variable "appartenance disciplinaire », en lien avec nos hypothèses. Certaines questions ont fait l'objet d'un recodage. Nous avons par exemple groupé les disciplines qui comptent peu d'enseignants : LV2 pour Espagnol et Allemand, Sciences expérimentales pour SVT, Sciences Physiques et Technologie, Lettres pour Français, Latin, Grec et Arts pour arts plastiques, éducation musicale. Mathématiques (Maths), Anglais et Histoire-Géographie (HG) sont restés indépendants.

Dans la ligne de l'investigation réflexive, les premières conclusions du questionnaire ont été présentées publiquement aux participants lors d'une réunion. Nous avons invité tous les enseignants sur le temps de la pause déjeuner, six d'en-

\footnotetext{
${ }^{5}$ BOEN spécial $n^{\circ} 11$ du 26 novembre 2015

http://www.education.gouv.fr/pid285/bulletin_officiel.html?cid_bo=95184

${ }^{6}$ Enseignements Pratiques Interdisciplinaires et Accompagnement personnalisé notamment. $B O E N n^{\circ} 22$ du 28 mai 2015 :

http://www.education.gouv.fr/pid25535/bulletin_officiel.html?cid_bo=89165
} 
tre eux ont pu venir : 2 professeurs de mathématiques, 2 professeurs d'anglais, 1 professeur de SVT et 1 professeur d'histoire géographie. Cette faible participation s'explique d'une part par le choix de l'horaire, de $13 \mathrm{~h} 15$ à $13 \mathrm{~h} 45$ pendant la pause méridienne et par le fait que les journées des professeurs sont déjà très chargées. Après une première partie sur le mode de l'exposé, les enseignants présents ont été invités à débattre et apporter des précisions afin d'enrichir notre analyse de leurs retours réflexifs. Cette réunion a été intégralement enregistrée et partiellement transcrite. Cet article présente les résultats croisés de ces deux recueils de données, questionnaire et transcription de la réunion.

Cette phase exploratoire s'appuie donc sur deux types de données: un questionnaire soumis à l'ensemble des enseignants du collège et une réunion de type focus group où les enseignants ont pu revenir sur les éléments saillants du questionnaire. Cela constitue la première étape d'une méthodologie qui ira ensuite vers un suivi individuel sur deux années. Les résultats présentés dans cet article concernent un petit nombre de cas, ils ne prétendent donc pas à une généralisation. Il s'agit de définir les caractéristiques d'un établissement en vue d'en faire une étude de cas (Passeron \& Revel, 2005).

Caractéristiques de l'échantillon

Le collège étudié se situe en zone rurale. Ses 41 professeurs accueillent 580 élèves de 10 à 16 ans. L'établissement est en phase d'expansion avec l'ouverture de deux classes de $6^{\mathrm{e}}$ à la rentrée 2015 et deux nouvelles classes à la rentrée 2016. L'enquête était ouverte en février 2015, pour une durée de 10 jours, et accessible en ligne via l'application LimeSurvey. Sur les 41 personnes contactées, 30 ont répondu au questionnaire, soit $73 \%$ du public visé. Il y a donc 11 personnes qui n'ont pas répondu. Les non-répondants sont majoritairement des hommes qui enseignent dans le domaine scientifique.

La médiane d'âge des répondants est de 38 ans avec un écart type de 9,06. Le premier quartile est à 35 ans. L'équipe semble plutôt stable avec une moyenne d'un peu plus de 6 ans d'ancienneté dans l'établissement. Si $63 \%$ sont en effet dans le même établissement depuis plus de 5 ans (19/30), dont $23 \%$ depuis plus de 10 ans, le tiers des répondants (9/30) effectuent leur première année au collège. Ces nouveaux venus sont répartis sur presque toutes les disciplines et cela a probablement modifié les équilibres en place. Sur le plan de l'expérience professionnelle, le premier quartile se situe à 11 ans, la médiane est à 14 ans et le $3^{\mathrm{e}}$ quartile à 20 ans d'ancienneté. L'écart type est de 10,6. Nous avons donc une population de praticiens chevronnés, qui, pour partie, a développé des habitudes de travail de longue date dans ce collège.

Les résultats présentés par la suite sont un préliminaire à notre recherche et ne sont pas généralisables. Cette phase exploratoire est, en effet, la première étape d'une méthodologie qui vise à établir un suivi de quelques acteurs sur le long terme. Une bonne connaissance du contexte dans lequel évoluent ces acteurs favorise une meilleure compréhension des logiques qu'ils mettent en œuvre dans leurs pratiques informationnelles.

\section{RESSOURCES MOBILISÉES PAR LES ENSEIGNANTS} POUR PRÉPARER ET FAIRE LA CLASSE

Un des objectifs de cette étude est d'identifier les ressources mobilisées par les enseignants à la fois pour préparer leurs cours, mais aussi pour faire la classe. Une très large majorité des enseignants interrogés déclare préférer réaliser eux- 
mêmes leurs cours (70 \% soit 21 sur 30). Les documents ainsi produits sont le résultat d'une création originale qui s'adapte parfaitement aux besoins de l'enseignant. Ce travail d'élaboration s'intègre dans une genèse documentaire (Gueudet $\&$ Trouche, 2010) qui se déploie sur un temps long. Les enseignants vont collecter, agréger et recomposer des ressources pour créer un nouveau document. Quels sont les outils et matériaux disponibles pour l'enseignant? En premier lieu le manuel scolaire, reste un élément essentiel du système de ressources des enseignants. Il est cependant de plus en plus concurrencé par les ressources en ligne, notamment les documents multimédias tels la vidéo.

\section{Le manuel scolaire,}

une hégémonie remise en cause

Dans la tradition française, le manuel scolaire occupe une place dominante depuis plusieurs générations. En effet, la naissance de l'édition scolaire est concomitante de celle de l'imprimerie (Chopin, 2008). Le livre scolaire est un rouage essentiel du système de ressource, il est la pièce maîtresse autour de laquelle se structure la documentation de l'enseignant. Le manuel scolaire propose un ensemble de ressources didactisées, c'est-à-dire des ressources qui ont été créées pour répondre à un objectif d'apprentissage. Il est découpé en différentes parties qui correspondent chacune à un contenu d'enseignement. Pour chaque partie, l'enseignant dispose de leçons et d'exercices autour desquels il bâtit son cours. Cet état de fait semble remis en cause par la révolution numérique en cours : les nombreuses ressources qui coexistent viennent se compléter pour répondre au mieux au besoin des enseignants (Pepin et al., 2013 ; Perrault, 2007 ; Rocha 2016).

Les usages des manuels sont très variables selon les disciplines, mais quelques lignes de force apparaissent dans notre enquête. Tout d'abord, les manuels scolaires sont surtout présents dans la phase de préparation des cours. Nous constatons que le manuel de la classe, donc celui dont les élèves disposent, n'est en aucun cas la seule ressource utilisée pour la préparation. Chez les $2 / 3$ des répondants, les manuels d'autres éditeurs sont aussi mis à profit. En plus des manuels papier que les enseignants possèdent chez $\operatorname{eux}^{7}$, ils sont un peu plus du tiers à signaler utiliser souvent des manuels en ligne. D'après ces déclarations, les manuels scolaires restent une ressource centrale dans la phase de préparation des cours, mais sous forme d'extraits, où l'enseignant puise le contenu qui lui paraît le plus pertinent, ou bien comme source d'inspiration. On note donc que les progressions élaborées par les auteurs des manuels ne sont pas utilisées comme telles, il y a bien un détournement de l'outil pour répondre au mieux au besoin de l'usager.

Pour ce qui est des usages en classe, le manuel semble moins présent. Seul 1/6 des enseignants indique utiliser le manuel pour tout le travail en classe. Dans la population étudiée, le manuel sert principalement à donner des exercices en classe $(47 \%$ soit $14 / 30)$ ou à la maison $(37 \%$ 11/30), très peu pour aborder une leçon $(23 \%$ soit $11 / 30)$. D'après les déclarations recueillies, le manuel est perçu comme un outil au service de l'enseignant. Les usages du manuel par les élèves ne sont pas évoqués, hormis pour la réalisation d'exercices.

Au-delà de ces tendances générales, nous observons de grandes variations selon les disciplines. Dans ce collège, les déclarations des professeurs d'anglais et de mathématiques sont en cela emblématiques de ces différences. En anglais, ce n'est pas le manuel distribué aux élèves qui est privilégié pour préparer les cours,

\footnotetext{
${ }^{7}$ Il s'agit des « spécimens » de manuel : envoi commercial des éditeurs aux enseignants lors d'un renouvellement du programme.
} 
mais plutôt les spécimens envoyés annuellement par les éditeurs. Cela s'explique en partie par le fait que les manuels de classe disponibles dans ce collège sont obsolètes et n'ont pas été changés depuis longtemps.

En revanche, chez les enseignants de mathématiques de cet établissement, le manuel de classe reste le document de référence aussi bien pour préparer les cours que pour travailler avec les élèves : $80 \%$ des professeurs (4/5) l'utilisent pour donner des exercices aux élèves. Ils déclarent également collaborer davantage pour discuter le choix d'un nouveau manuel (50\% contre $20 \%$ en anglais). D'ailleurs, face à l'ouverture de deux classes en $6^{\mathrm{e}}$ et l'impossibilité de doter tous les élèves du manuel déjà en service, l'équipe n'a pas hésité à se tourner vers une solution numérique gratuite : le manuel Sésamath. Cofronté au même problème, les enseignants d'anglais ont choisi de ne pas distribuer de manuels aux élèves de $6^{\mathrm{e}}$.

En dehors des contingences matérielles, ces différences dans l'usage du manuel de classe conduisent nécessairement les enseignants à élaborer des dispositifs didactiques différents. Comment les professeurs d'anglais pallient-ils l'absence d'un manuel de classe qui leur convient? Pourquoi ne ressentent-ils pas le besoin d'un référent commun alors qu'en mathématiques cela semble faire partie de leur culture ? Autant de questions qui seront peut-être élucidées lors de la réalisation des entretiens.

Des ressources en provenance d'Internet

de plus en plus présentes

En parallèle des manuels scolaires, les enseignants de ce collège ont recours à de nombreuses ressources multimédias. Un tiers des enseignants indiquent préparer leur cours à partir d'un logiciel spécialisé et surtout, $63 \%$ (19/30) des personnes interrogées font une recherche en ligne durant la phase de préparation de leur cour. Une partie de l'attrait du numérique tient au fait que de nombreuses ressources multimédias sont accessibles librement. La vidéo est un support particulièrement prisé : $57 \%(17 / 30)$ déclarent utiliser souvent la vidéo en classe. Ce fait est tout particulièrement avéré en langues, ainsi qu'en sciences humaines et expérimentales. Cela suppose un temps préalable de recherche et de sélection ainsi que la préparation d'un document d'exploitation du support vidéo. Les images fixes sont aussi très présentes, toutes disciplines confondues $(80 \%$ des enquêtés soit 24/30).

Les supports utilisés pour stocker les ressources et les documents sont révélateurs de la présence des ressources numériques : la quasi-totalité des répondants (29/30) utilise leurs ordinateurs personnels. La clé USB permet de faire le transfert du domicile vers le collège pour $86,7 \%$ des enseignants interrogés (soit 26 sur 30). Le support qui arrive en $3^{\mathrm{e}}$ position est plus surprenant puisqu'il s'agit du classeur. Questionnés à ce sujet, plusieurs enseignants ont expliqué imprimer les documents à des fins de sauvegarde et pour disposer d'une version papier accessible en classe à tout moment. Si les documents orignaux sont bien au format numérique, les documents distribués aux élèves restent ancrés dans l'univers papier.

Il apparaît donc que les phases de recherche de ressources et de création de documents se déroulent principalement via les supports numériques. Ce fait est confirmé par l'utilisation d'Internet pour $90 \%$ d'entre eux en cas de besoin d'information. Quels sont les sites web utilisés par les enseignants pour rechercher de nouvelles ressources ? Le recours à un moteur de recherche généraliste est majoritaire, mais certains critères émergent quant aux choix des sites sélectionnés dans la liste des résultats : les enseignants utilisent essentiellement des ressources élabo- 
rées par d'autres collègues. Ils sélectionnent en priorité des sites web ou des blogs tenus par des enseignants ( $73 \%$ soit $22 / 30$ ), puis des sites web disciplinaires des académies (57\% soit 17/30), et enfin des sites web d'associations professionnelles (40 \% soit 12/30). Dans les trois cas, ce sont d'autres enseignants qui gèrent les sites et publient des ressources. Nous sommes donc face à une communauté d'enseignants qui va puiser dans un réservoir de ressources produites par d'autres enseignants.

À l'opposé, les ressources du centre de documentation et d'information (CDI) de l'établissement sont désertées : $56 \%$ (soit 17/30) des enseignants vont rarement au CDI en cas de besoin d'information et $30 \%$ jamais (soit 9 sur 30). La complexité du système d'information et la dépersonnalisation du catalogue informatique peuvent être une explication à cette désaffection marquée. Diekema et Olsen (2012) font clairement le lien entre la sous-utilisation des bibliothèques scolaires et le système de recommandation par les pairs qui a la préférence des enseignants. Dans une étude portant sur une école primaire, Beguin-Verbrugge et Desprets-Lonnet (2011) notent cette même préférence pour des ressources recommandées par les pairs et une $\mathrm{BCD}$ (bibliothèque centre de documentation) dont l'usage est pensé exclusivement en direction des élèves.

Si les enseignants interrogés utilisent massivement des ressources produites par leurs pairs, ils sont peu nombreux à publier eux-mêmes leurs ressources sur Internet (13\% soit 4 sur 30), et, pour les professeurs concernés, un seul met réellement à disposition des documents à destination d'autres professeurs de manière publique sur le site académique. Pour les 3 autres, il s'agit d'une mise à disposition en direction des élèves via l'ENT du collège.

Si nous nous focalisons maintenant sur les professeurs d'anglais et de mathématiques, nous constatons que les enseignants des deux disciplines déclarent utiliser fréquemment des sites web disciplinaires. En revanche, les professeurs d'anglais ont plus souvent recours aux sites web généralistes, c'est-à-dire des sites qui ne sont pas à finalité éducative. Le besoin de ressources authentiques, préconisées par les programmes, explique sans doute cette différence. Ce résultat est aussi à croiser avec une déclaration d'usage plus fréquent de la vidéo et de l'audio en anglais qu'en mathématiques.

Dans cet établissement, les enseignants ont intégré dans leur palette une multitude de ressources qui dépasse largement le cadre du manuel scolaire. Même s'il garde une place de choix, le manuel est complété par les nombreuses ressources disponibles en ligne librement. Les manuels numériques sont utilisés pour préparer les cours, mais ne sont pas pensés pour faire la classe ou comme soutien aux élèves au domicile. L'environnement clos du manuel numérique «entre en conflit avec les activités des enseignants qui se caractérisent par la (re)conception de documents à partir d'un large éventail de ressources. » (Beauné, 2013) En outre, ils représentent un coût important : les licences numériques doublent l'achat d'une version papier et sont limitées dans le temps. Pour l'heure, il semblerait donc que les manuels numériques ne correspondent pas aux attentes des enseignants.

$\mathrm{Au}$ terme de cette partie, nous pouvons éclairer deux de nos hypothèses. Tout d'abord (H1), «de plus en plus de ressources numériques sont utilisées en complément des manuels scolaires », semble confirmée par les déclarations des enseignants de ce collège. La palette de ressources mobilisées pour faire la classe s'est considérablement enrichie, l'image sous toutes ses formes (fixe, vidéo, mais aussi dynamique via les logiciels) est beaucoup plus présente ainsi que, dans une moindre mesure, l'utilisation du son (essentiellement en langues et éducation mu- 
sicale). Les enseignants préfèrent très majoritairement concevoir leurs propres documents, ils combinent différentes ressources numériques ou papier afin de répondre au mieux à leurs besoins. La dimension de conception - design- est prépondérante : 21 sur 30 préfèrent réaliser eux-mêmes leurs supports de cours et ce résultat semble indépendant de leur ancienneté professionnelle. La plasticité des documents numériques a sans doute renforcé cette tendance de fond. L'équipement en vidéoprojecteur et en ordinateurs de la totalité des salles de l'établissement depuis quelques années a probablement été un élément facilitateur de l'introduction des ressources numériques multimédias en cours. Il conviendra, dans le suivi individualisé des enseignants, de questionner, d'une part, ce que les documents multimédias apportent aux enseignants, mais également, d'autre part, d'étudier quelles compétences ils ont développées ou renforcées par la manipulation de ces ressources.

L'hypothèse $(\mathrm{H} 2)$, «la recherche d'informations sur Internet est devenue une pratique régulière dans le cadre professionnel », est totalement confirmée. Nous aurions souhaité faire jouer les variables de l'âge et de l'ancienneté pour préciser cette hypothèse, mais le faible échantillon ne permet pas d'apprécier un lien éventuel entre ces variables et la pratique d'Internet. En revanche, il ne fait aucun doute que, pour la préparation des cours, Internet est un outil majeur. Nous notons particulièrement un usage fréquent des sites web disciplinaires académiques ou développés de manière autonome par des enseignants. Ce résultat important ouvre sur la dimension collective du travail documentaire via la mutualisation des ressources de préparation de cours.

\section{DIMENSIONS COLLECTIVES DU TRAVAIL DOCUMENTAIRE DANS LES ÉQUIPES DISCIPLINAIRES}

Le travail collectif est une composante essentielle du métier d'enseignant, car, bien qu'il soit le plus souvent seul face aux élèves, dans l'établissement scolaire, l'enseignant est pris dans un faisceau d'équipes. Dans le rapport L'insertion subjective d'enseignants novices sous la direction d'Amendola et al., Philippe Losego (2015 : 60) distingue 3 formes principales de travail collectif en fonction de leur impact sur la forme scolaire : les échanges verbaux, les échanges de matériaux et les préparations conjointes. Nous nous appuyons sur ce cadre pour mener notre analyse, mais au préalable une clarification sémantique s'impose.

Dans cette partie, nous allons aborder la dimension collective du travail documentaire. C'est surtout sur ce point qu'ont porté les échanges lors de la réunion de présentation des résultats aux acteurs du terrain. Dans le questionnaire, ce sont les termes généraux de « collaboration » ou le verbe «collaborer » qui ont été retenus pour la formulation des questions, sans autre précision. Lors de la réunion, un consensus s'est rapidement dégagé pour définir la collaboration comme un processus formel de co-construction d'une ressource alors que le terme «échanger » renvoie à la mise à disposition d'un produit fini et revêt un caractère fortuit.

«-Henriette (HG) : Collaborer, tu construis ensemble.

- Marie (Math) : Échanger, tu échanges un truc que t'as fait perso.

- Henriette : Et échanger, tu donnes un produit fini.» (27'49" à 28'07”)

Cela correspond à la définition que donne Marcel et al. (2007: 10) : «la collaboration se caractérise d'abord par l'interdépendance engendrée par le partage d'un espace et d'un temps de travail comme par le partage de ressources ». 
Marie (Maths) conforte cette distinction entre collaborer et échanger :

"Voilà le brevet blanc en $4^{e}$ ou le brevet en $3^{e}($...) ça c'est des trucs on se mettait tous ensemble autour de la table et on le définissait et après, le reste du temps s'est souvent $d u$ travail perso fait par les enseignants et il dit à l'autre bah tiens si ça t'intéresse j'ai fait ça, ça a bien marché ou est-ce que tu as un truc là-dessus. » (16').

Les collègues d'une même discipline sont souvent les premiers collaborateurs : seuls $20 \%$ (6 décembre) des enseignants questionnés déclarent ne jamais collaborer avec les collègues de leur discipline ${ }^{8}$ tandis que $40 \%(12 / 30)$ déclarent des collaborations fréquentes.

\section{Échanges verbaux}

Le premier échelon de la typologie proposée par Losego se situe au niveau de l'échange verbal, que ce soit pour parler des élèves ou demander des conseils. Dans notre enquête, nous avons questionné les enseignants sur leur réaction face à un manque de ressources. Les avis sont partagés : $57 \%$ déclarent demander rarement ou jamais à un collègue (13/30) contre $40 \%$ qui déclarent demander souvent ou toujours à un collègue (12/30). Lorsque l'on croise ce résultat avec le filtre disciplinaire, il apparaît que, dans cet établissement, les enseignants de langues n'adhèrent pas à cette pratique, alors qu'elle est régulière en mathématiques et en histoire géographie.

En revanche, les échanges informels concernant une question de la discipline sont très fréquents : pour les $2 / 3$ des répondants, ils se produisent souvent ou toujours.

Du point de vue disciplinaire, nous constatons une différence importante entre les professeurs de mathématiques et d'anglais. Si pour les premiers, échanger sur les problèmes de la discipline est courant, il semble que, dans l'équipe d'anglais, cela soit beaucoup plus rare. D'après leurs déclarations, nous concluons que les enseignants de ce collège communiquent plus souvent pour discuter de ce qui concerne la discipline que pour demander des ressources.

Échange de matériaux

Le second niveau de travail collectif identifié par Losego concerne l'échange de matériau pédagogique. Cette pratique est répandue, mais peu intense dans le collège étudié : $30 \%$ des enseignants (9/30) signalent échanger souvent ou toujours des ressources avec leurs collègues contre $53 \%$ qui indiquent échanger rarement.

En appliquant le filtre disciplinaire, nous remarquons que les champs disciplinaires où s'échangent le plus de matériaux sont l'histoire géographie, les mathématiques et l'espagnol. Là encore, les professeurs d'anglais sont moins présents. Les disparités dans une même discipline peuvent sans doute s'expliquer par un déséquilibre entre ceux qui donnent et ceux qui reçoivent. On peut supposer que les destinataires des documents n'ont pas déclaré échanger des ressources s'ils n'en donnent pas eux-mêmes.

La stabilité des équipes peut aussi être un facteur important comme en témoignent Stéphane (SVT) et Henriette (HG) :

Stéphane (SVT) : "Après là ça va être la première année où il va y avoir une collègue où on sait qu'elle va rester. Avec Céline j’échangeais des trucs aussi, ponctuelle-

\footnotetext{
${ }^{8}$ A noter que pour certaine discipline comme l'éducation musicale, l'équipe est inexistante.
} 
ment, sur des activités qui fonctionnent bien, mais à mon avis on va bosser plus encore avec Sandrine vu que maintenant elle est stable dans l'établissement. » (19'30”)

Henriette (HG) : "Je pense qu'après on le fera avec Hortense. Le fait qu'elle n'était pas là, et elle était aussi dans l'agrég les années précédentes, c'est aussi pour ça qu'on l'a pas fait avec elle. » (17'00”)

Le même type d'échange avec des collègues de la même discipline, mais d'un autre établissement montre que des collaborations existent (55\% des enseignants interrogés soit 17/30). Ces échanges sont cependant moins fréquents qu'au sein de l'établissement d'exercice. On peut faire l'hypothèse qu'ils sont tributaires des liens d'amitié qui se sont créés soit lors de la période de formation initiale, soit lors de passages dans d'autres établissements.

\section{Préparation commune}

Le troisième niveau de travail collectif, qui impacte davantage la forme scolaire, concerne les préparations conjointes, c'est-à-dire la réalisation commune d'un document de travail ou d'une progression de séquence. Les évaluations communes sont un contexte favorable à ce type de collaboration : $23 \%$ des enseignants déclarent une pratique régulière, essentiellement en histoire-géographie et en Lettres, sans doute à cause des épreuves de brevet blanc à préparer comme le confirme Marie (Maths) :

"Préparer par exemple le brevet blanc on va s'y coller donc soit par mail, soit autour de la table ou les deux souvent. On échange pour définir les exercices qu'on va mettre, modifier les énoncés en fonction des choix d'exercices » (15'30").

Concernant les préparations de séances ponctuelles ou la mise en place d'une progression, il y a respectivement $66 \%$ et $70 \%$ des enseignants interrogés qui répondent positivement. Mais la pratique régulière (toujours ou souvent) concerne seulement $30 \%(9 / 30)$ des répondants pour la mise en place d'une progression et $23 \%(7 / 30)$ pour la préparation de séance ponctuelle.

À noter que les enseignants de mathématiques et d'histoire-géographie sont ceux qui ont le plus développé cette forme de travail collectif. Lors de la réunion de présentation, Henriette (HG) a expliqué que, depuis plusieurs années, elle préparait tous ses nouveaux cours avec une collègue allant même jusqu'à déclarer :

"Mais Hélène et moi, en 5e, on a quasiment les mêmes cours. Les mêmes traces écrites, on définit beaucoup de choses ensemble. En fait nos meilleurs cours, on les fait ensemble. Donc je pense que du coup l'année prochaine, comme on devra refaire les programmes, toutes les trois, on bossera vraiment ensemble. A priori on aura les mêmes cours. C'est vachement bien pour le suivi des élèves. » (17'35")

De la même manière, Marie a indiqué qu'en mathématiques, l'équipe élabore une progression commune sur l'année, à la semaine près, pour tous les niveaux, alors que dans les autres disciplines, les préparations conjointes restent une forme marginale du travail collectif. Les préparations communes sont toujours centrées sur un projet précis : une épreuve commune comme évoquée plus haut, mais aussi le travail en groupe de compétences. Il ne s'agit pas toujours de l'élaboration de ressources en commun, cela peut se limiter à la détermination de cadre commun pour mettre en œuvre une activité.

- Amy (Anglais) : "Les échanges, c'est très informel, tiens regarde ce que j'ai fait oh bah tiens oh bah moi j'ai fait ça, mais y a rien de, c'est pas du tout formalisé. À part l'heure de groupe de compétences où il y a une concertation 2 ou 3 fois par an». 
La discipline qui semble la plus active sur le plan de l'échange des ressources et les préparations conjointes est l'histoire géographie, c'est aussi une équipe où il n'y a pas eu de modification dans la composition depuis 5 ans. En mathématiques, le travail d'équipe est aussi très présent, alors même que l'équipe s'est profondément renouvelée les 5 dernières années. Les professeurs de mathématiques déclarent travailler davantage ensemble pour mettre en place une progression commune. En parallèle, ils signalent tous échanger des ressources ou préparer des séances ensemble, mais à des fréquences moindres. On peut donc supposer qu'un effort est fait pour mettre en place une cohérence didactique dans cette discipline à l'échelle de l'établissement, ce qu'a confirmé Marie lors de la réunion de présentation. En anglais, les professeurs manifestent une fréquence de collaboration infime, seuls deux enseignants signalent collaborer souvent, tandis que les trois autres collaborent rarement ou de temps en temps. L'équipe semble scindée en 2 groupes. À noter également que l'équipe est jeune puisque 2 nouvelles personnes sont arrivées cette année dont l'une est sur 2 établissements.

Nous pouvons donc établir que, pour ce collège, le travail collectif disciplinaire déclaré existe de manière ponctuelle. C'est surtout au niveau des échanges verbaux qu'il est le plus développé. L'échange de ressources pédagogiques et les préparations conjointes sont le fait d'un petit nombre de personnes et surtout de situation spécifique. D'après les déclarations recueillies, la collaboration s'exerce sur un temps défini et en vue de réaliser un objectif précis. Le renouvellement des programmes peut être un facteur catalyseur des collaborations disciplinaires. Lors de la réunion, plusieurs acteurs ont indiqué que la logique par cycle et la rapidité de mise en œuvre rendaient quasi-obligatoire une répartition de la charge de travail par les membres des équipes. De plus, la mise en œuvre des enseignements pratiques interdisciplinaires (EPI) à la rentrée 2016 devrait contribuer à développer le travail collectif au niveau interdisciplinaire.

\section{DIMENSIONS COLLECTIVES DU TRAVAIL DOCUMENTAIRE EN INTERDISCIPLINARITÉ}

Une des caractéristiques principales du travail interdisciplinaire est la démarche de projet, or, cette situation cadrée est propice au développement de collaborations. Le travail interdisciplinaire est plutôt bien implanté dans ce collège où de nombreux projets sont menés : $80 \%$ des sondés déclarent avoir travaillé au moins une fois avec un collègue d'une autre discipline depuis le début de l'année scolaire (24/30). Nous verrons d'abord en quoi la dimension collective du travail interdisciplinaire est intimement liée à la notion de projet puis nous analyserons les échanges de ressources qui ont lieu dans ce cadre.

\section{Travail en projet}

Nous avons également questionné les enseignants pour savoir «quelles sont les thématiques de collaborations les plus fréquentes avec [leurs] collègues, quelle que soit leur discipline ? » (Figure 1). D’après les déclarations des répondants, un des thèmes de prédilections pour les collaborations est donc les projets, mais aussi les réflexions concernant la profession au sens large. Nous constatons une très forte mobilisation : $86 \%$ des enseignants répondent favorablement à l'existence de collaboration sur des projets (26/30). Toutes les disciplines sont impliquées, mais lorsque nous regardons les fréquences, seules quelques disciplines, Anglais, Lettres, Sciences expérimentales et Histoire-Géographie ont développé des pratiques régulières de collaboration sur projet. 
Figure 1 : Quelles sont les thématiques de collaborations les plus fréquentes avec vos collègues, quelle que soit leur discipline?

\begin{tabular}{|c|c|c|c|c|c|c|c|c|}
\hline & $\begin{array}{c}\text { Maths } \\
5 / 5\end{array}$ & $\begin{array}{c}\text { Anglais } \\
5 / 5\end{array}$ & $\begin{array}{c}\text { Arts } \\
2 / 2\end{array}$ & $\begin{array}{l}\text { Lettres } \\
5 / 5\end{array}$ & $\begin{array}{c}\mathrm{HG} \\
\mathrm{EMC} \\
4 / 4\end{array}$ & $\begin{array}{c}\mathrm{LV} 2 \\
3 / 3\end{array}$ & $\begin{array}{c}\text { Sc. } \\
\text { Exp. } \\
4 / 4\end{array}$ & Moyenne \\
\hline $\begin{array}{l}\text { Préparation contrôle } \\
\text { commun }\end{array}$ & 4 & 0 & 0 & 5 & 4 & 3 & 1 & $58 \%$ \\
\hline $\begin{array}{l}\text { Système d'évaluation } \\
\text { des élèves }\end{array}$ & 5 & 3 & 1 & 4 & 3 & 3 & 3 & $77 \%$ \\
\hline $\begin{array}{l}\text { Mise en place d'une } \\
\text { progression }\end{array}$ & 5 & 2 & 1 & 4 & 3 & 2 & 2 & $66 \%$ \\
\hline $\begin{array}{l}\text { Préparation d'une } \\
\text { séance }\end{array}$ & 5 & 2 & 0 & 4 & 3 & 3 & 3 & $67 \%$ \\
\hline $\begin{array}{l}\text { Projet de classe ou de } \\
\text { niveau }\end{array}$ & 4 & 2 & 1 & 5 & 4 & 3 & 3 & $86 \%$ \\
\hline Organisation de l'équipe & 4 & 4 & 1 & 5 & 3 & 3 & 4 & $83 \%$ \\
\hline Choix d'un manuel & 4 & 4 & 0 & 4 & 4 & 2 & 3 & $69 \%$ \\
\hline $\begin{array}{l}\text { Réflexion sur un pro- } \\
\text { blème de la discipline }\end{array}$ & 5 & 3 & 1 & 5 & 4 & 3 & 4 & $87 \%$ \\
\hline $\begin{array}{l}\text { Réflexion sur une ques- } \\
\text { tion de la profession }\end{array}$ & 3 & 5 & 1 & 5 & 4 & 3 & 4 & $87 \%$ \\
\hline
\end{tabular}

Le propos est à nuancer, car les éléments de réponses ne permettent pas de distinguer s'il s'agit de projets disciplinaires ou interdisciplinaires. Comme les collaborations en anglais sont faibles à l'intérieur de la discipline, on peut supposer que le travail en projet revêt davantage une dimension interdisciplinaire. À l'inverse, en mathématiques où la collaboration disciplinaire est forte, la dimension de travail en projet interdisciplinaire est peu présente, ce que confirme Marie dans ses déclarations :

"Le fait qu'on ait une progression commune très forte en math, sur tous les niveaux, c'est vachement compliqué de justement travailler en interdisciplinarité parce que pour que ce soit pertinent et cohérent, il faut effectivement qu'il y ait une progression avec, justement, la matière avec qui tu bosses. » Marie (Math - 25' 38”).

Cet impératif d'une progression commune, c'est-à-dire d'un cadre de travail défini dans le temps avec des objectifs précis est posé comme une condition de la collaboration interdisciplinaire : travailler en projet suppose «avoir une progression commune sur le même thème et du coup après on se dispatche qui peut faire quoi dans sa langue et puis avoir un travail final commun » Amélie (Anglais - 24'31").

Au-delà d'un objet d'étude commun, le travail interdisciplinaire peut amener à des ajustements sémantiques : «J'avais discuté avec Malika [professeur de mathématiques] en $5^{e}$ et on s'était rendu compte qu'on n'utilisait pas forcément tout le temps les mêmes termes pour décrire l'évolution d'une courbe. Du coup on s'était mis d'accord» Stéphane (SVT - 24' 50").

Les échanges qui ont eu lieu pendant la réunion confirment, pour les enseignants présents, que le travail interdisciplinaire tient une place importante dans leurs approches professionnelles, mais cela n'aboutit pas nécessairement à un échange de matériaux didactiques. 


\section{Échange de ressources}

Une question portait explicitement sur l'échange de ressources ou la préparation conjointe de séances avec un professeur d'une autre discipline. Cette pratique est minoritaire avec $20 \%$ d'échanges réguliers (6/30) contre $73 \%$ (22/30) d'échanges rares ou inexistants. Mais, parmi les répondants, nous retrouvons un enseignant d'anglais et les deux professeurs d'espagnol. Pendant la réunion, Henriette (HG) a avancé une explication à ce phénomène : selon elle, le travail en projet interdisciplinaire convoque des points de vue différents autour de mêmes objets d'étude, chaque discipline apporte alors ses propres ressources qui permettent d'éclairer le point de vue de la discipline. L'ensemble des participants étaient d'accord avec cette opinion, sauf pour le travail en histoires des arts où la mutualisation des ressources est plus évidente.

Les réponses au questionnaire indiquent en effet que le partage de ressources est ponctuel en lettres, en anglais, en espagnol et en histoire géographie. En mathématiques, il est quasi inexistant. Marie (Maths) indiquait lors de la réunion de présentation que la progression commune ne donnait pas la souplesse nécessaire pour s'adapter aux contraintes inhérentes aux disciplines partenaires.

D'après les réponses, le travail en interdisciplinarité semble bien implanté dans cet établissement. Cependant, les collaborations semblent concerner essentiellement le travail par projet. Les échanges de ressources sont marginaux, car ils ne sont pas, pour les enseignants interrogés, nécessaires à la mise en œuvre de telles collaborations. L'introduction des Enseignements Pratiques Interdisciplinaires (EPI) à la rentrée 2016 peut faire évoluer les choses. Si les ressources de travail pour la classe ne sont pas ressenties comme un besoin, des ressources communes pour le suivi et l'évaluation des élèves devront nécessairement être créées.

\section{SYNTHÈSE ET PERSPECTIVES}

Un des objectifs de cette étude est de dresser une cartographie des pratiques informationnelles des enseignants d'un collège, c'est-à-dire de déterminer à la fois les matériaux, les sources d'information, les stratégies de recherche des enseignants, mais aussi les collaborations qui existent dans une même équipe disciplinaire ou entre des enseignants issus de différentes disciplines.

Concernant la manière de travailler de ces enseignants, la partie conception de cours tient une place prépondérante. Les contenus d'enseignements sont agencés en combinant plusieurs types de documents : des éléments textuels comme des extraits de différents manuels, des éléments iconographiques, de l'audiovisuel, des éléments sonores. Ceci est confirmé par le livrable 2.1 du projet RéVEA (Khaneboubi, 2014) qui mentionne une grande variété des ressources mobilisées par les enseignants dans toutes les disciplines étudiées. En dehors des ressources marchandes - éditeurs scolaires - les enseignants de ce collège tendent à privilégier les ressources validées par les pairs: site d'enseignants, sites académiques ou d'associations professionnelles sont en effet les réponses les plus citées dans les déclarations. Le système de recommandation par les paires semble donc bien être une constante.

Nous pouvons qualifier les enseignants de «bricoleurs » dans le sens qu'en donne Levi-Strauss dans La pensée sauvage (1960:27) : "la règle de son jeu est de toujours s'arranger avec les "moyens du bord», c'est-à-dire un ensemble à chaque instant fini d'outils et de matériaux, hétéroclites au surplus, parce que la composition de l'ensemble n'est pas en rapport avec le projet du moment, ni d'ailleurs avec aucun projet particulier, mais est le résultat contingent de toutes 
les occasions qui se sont présentées de renouveler ou d'enrichir le stock, ou de l'entretenir avec les résidus de constructions et de destructions antérieures ». Perrenoud (1983 : 12) notait également que «dans beaucoup de cas, ce n'est pas d'abord la nécessité économique qui pousse au bricolage, mais la part de création qu'il permet, le défi supplémentaire qui consiste à parvenir à ses fins “ avec les moyens $d u$ bord " ». Cette propension au «bricolage » est renforcée par le développement du numérique (Moiraud, 2009 ; Messaoui, 2014). Il en effet devenu très facile de copier, stocker, modifier et éditer des documents numérisés. C'est ce qui se joue dans 1'instrumentalisation (Rabardel, 1995). Si nous considérons le manuel scolaire comme un artefact. (Rabardel 1995), les auteurs ont soigneusement choisi les différents documents qui le composent dans une logique de progression. L'enseignant va sélectionner quelques éléments qu'il va associer à d'autres éléments conçus par lui ou trouvés ailleurs. L'usager va détourner la progression prévue dans le manuel de sa fonction initiale pour l'adapter à son propre usage, sa propre logique.

Les premiers portraits d'enseignants qui ont été présentés dans le livrable 3.1 du projet ReVEA (Gueudet, 2015) tendent à confirmer les éléments présentés dans cet article : les enseignants d'anglais ont tendance à utiliser davantage de ressources authentiques que de ressources didactisées et privilégient la recherche de ressources vidéo via notamment le moteur de recherche intégré à YouTube. Les auteurs notent également l'utilisation de plusieurs manuels «davantage, pour les professeurs d'anglais, une inspiration pour la structuration des séquences, pour les grandes idées thématiques, pour les exemples de textes ou d'images à travailler, qu'un support systématique associé aux activités menées en classe. »(Gueudet, 2015 : 6). Dans ce même livrable, les auteurs constatent qu'en mathématiques le manuel continue d'occuper une place centrale et que les ressources didactisées sont les plus mobilisées. Des tendances par discipline semblent donc se dégager. Elles seront à corroborer dans les suites de notre étude.

La dimension collective du travail documentaire recouvre des disparités importantes selon les disciplines. Même si certaines équipes disciplinaires sont plus actives que d'autres, cela semble essentiellement dû au nombre de personnes qui composent l'équipe et à leur stabilité contrairement à ce qui était évoqué dans notre hypothèse $\mathrm{H} 4$ : «La discipline d'enseignement a une influence sur l'intensité des échanges de ressources ». En revanche, les déclarations des enseignants semblent soutenir le fait que ceux qui partagent le plus leurs ressources sont aussi ceux qui travaillent le plus en équipe disciplinaire ou interdisciplinaire et inversement. Les profils des équipes de mathématiques et d'anglais sont en cela très contrastés, que ce soit sur l'aspect des collaborations disciplinaires, des collaborations interdisciplinaires ou encore sur l'utilisation du manuel scolaire. Là encore, les auteurs du livrable 3.1 pointent les mêmes différences entre les pratiques des professeurs d'anglais et ceux de mathématiques. De part et d'autre une conception de document a bien lieu : chez les enseignants de mathématiques, il s'agit davantage d'une recomposition de ressources didactisées alors que les enseignants d'anglais ont tendance à créer un document d'accompagnement pédagogique d'une ressource authentique. Comprendre les raisons de ces spécificités disciplinaires est un enjeu majeur pour la suite de l'étude. Ces premiers résultats renforcent le choix d'étudier conjointement la dynamique des deux disciplines, anglais et mathématiques. Pouvons-nous dire que, dans un cas comme dans l'autre, les acteurs mettent en œuvre un processus de redocumentarisation (Pédauque, 2006), c'est-à-dire qu'ils créent non seulement des documents originaux à partir de ressources combinées, mais également des collections de documents avec ce que cela suppose de 
traitement documentaire (résumé, ajout de métadonnées...) ? C'est une dimension qu'il nous faudra investir dans la suite de cette étude.

Enfin, sur le plan des pratiques informationnelles, l'utilisation régulière d'Internet est très ancrée, et cela, quel que soit l'âge des enseignants (H2). L'accroissement du recours aux ressources numériques bouleverse la place hégémonique que tenait traditionnellement le manuel scolaire dans le système français. La souplesse offerte par les ressources numériques tend à même à faciliter la reconception de ressources pour la classe, à condition bien sûr d'avoir une maîtrise technique suffisante des outils. Nous avons constaté que les enseignants recherchent principalement des ressources déjà élaborées, mais le plus souvent, il s'agit d'une base qu'ils vont modifier, adapter, transformer y compris les débutants contrairement à notre hypothèse $\mathrm{H} 3$. Cette genèse documentaire est à la fois un processus d'appropriation du contenu, mais aussi un processus de remise en forme. Tout l'enjeu de notre étude est justement de comprendre comment ce travail de reconception s'élabore, quels sont les facteurs qui guident et influencent les choix des enseignants? En soi, le travail documentaire est un procédé de réécriture qui met en jeu des compétences de recherche et de sélection de l'information, des connaissances professionnelles didactiques et pédagogiques ainsi que des compétences techniques. Ces trois ensembles de compétences correspondent au champ étudié par la translittératie, dont «le préfixe «trans- » suggère à la fois la quête de transversalité dans les démarches de recherche, d'appropriation et de réécriture de l'information, mais également, la capacité de chacun à transférer dans divers environnements et contextes informationnels et techniques des acquisitions antérieures. » (Delmotte et al., $2014: 1$ )

Un axe déterminant pour la poursuite du travail sera la définition des facteurs qui président à la sélection et à l'organisation d'une séquence de cours. Une première classification des facteurs est proposée dans le livrable 5.2 du projet RéVEA (Bento, 2016). Les auteurs distinguent trois classes de facteurs pour lesquels il est possible d'identifier plusieurs critères. La première concerne les facteurs individuels et relationnels. Ils sont spécifiques au vécu de l'enseignant, à ses goûts et aux réseaux dans lesquels il s'inscrit. La seconde relève des facteurs didactiques et pédagogiques : analyse de la situation de classe, niveaux et intérêt des élèves, objectifs assignés à la leçon ou encore préconisations institutionnelles. La troisième classe touche aux facteurs contextuels qui regroupent le critère matériel, le critère marchand et le critère territorial. Cette proposition reprend les trois dimensions éthiques, économiques et identitaires qui sont associées aux pratiques informationnelles. Cependant, notre problématique s'intéresse aussi à l'expertise documentaire, c'est-à-dire à l'influence des compétences des enseignants dans la recherche, la sélection et le traitement des informations dans le cadre du travail documentaire. Il conviendra pour la suite de définir précisément ce que recouvre l'expertise documentaire (Wang, 2016). Au vu des premiers éléments collectés ici, cela semble aller au-delà d'un savoir-faire technique. Il s'agit plutôt d'une expertise qui combinerait culture informationnelle, culture numérique et culture didactique.

L'enquête par questionnaire a permis d'avoir une vue d'ensemble des pratiques informationnelles d'une majorité des enseignants d'un collège, malgré cela, de nombreuses questions restent en suspens. Les enseignants interrogés utilisent de préférence des ressources élaborées par d'autres enseignants et connaissent les sites où elles sont disponibles. Cependant, ils utilisent très majoritairement un moteur de recherche généraliste pour y accéder. Comment expliquer ce comportement? Autres questions liées spécifiquement aux contrastes entre l'anglais et les 
mathématiques : comment les professeurs d'anglais pallient-ils l'absence d'un manuel de classe qui leur convienne? Pourquoi ne ressentent-ils pas le besoin d'un référent commun alors qu'en mathématiques cela semble faire partie de la culture professionnelle des enseignants ? Quel impact aura la mise en place de la réforme avec, notamment l'organisation des programmes en cycle de 3 ans et les EPI, sur le travail collectif des enseignants? Enfin, quels liens peuvent exister entre l'expertise documentaire des enseignants et les médiations documentaires qu'ils mettent en œuvre en direction des élèves ? La poursuite de l'étude par des entretiens et des observations avec les professeurs d'anglais et de mathématiques sera l'opportunité de collecter de nouveaux éléments qui permettront de caractériser davantage le travail documentaire de ces enseignants, d'identifier les pratiques informationnelles en jeu et leur impact sur la mise en œuvre de l'éducation aux médias et à l'information.

Anita MESSAOUI

S2HEP - Educ'Tice ENS Lyon

\begin{abstract}
The documentational approach to didactics (Gueudet and Trouche, 2010) highlights the importance of working on resources for teachers. This work is completely changed by digital environments that are developed over the past ten years. This study takes part in the ANR-REVEA research project. It focuses specifically on the documentary work of Mathematics and English teachers. The article proposes to study the documentary practices of teachers in a rural secondary school by identifying at first what are the resources used by teachers to prepare and to do their teaching. The investigation then brings elements on the collective dimension of the documentary work of teachers of this school, in the same subject teams, but also in the context of interdisciplinary work. Finally, these first elements will be put into perspective in light of deliverables already published by the actors of the ReVEA project.

Keywords : documentary practices, documentational approach to didactics, teaching resources, collective teacher work, documentary work, online information seeking, mathematics teaching, English teaching
\end{abstract}

\title{
Bibliographie
}

Alava S. (1997) «Pratiques documentaires des enseignants et processus de professionnalisation lire pour enseigner »-Spirale 19 (89-100).

Amendola C., André B., \& Losego P. (2015) L'insertion subjective d'enseignantes novices. Le cas de l'enseignement primaire dans le canton de Vaud. Lausanne : HEP Vaud.

https://www.hepl.ch/files/live/sites/systemsite/files/ueragirs/th\%c3\%a9matiques\%20AGIRS/Insertion\%20professionnelle/Rapport_INSERS UB_nov_15.pdf

Beauné A. (2013) «Manuels numériques, TICE au collège» - Adjectif. net. http://www.adjectif.net/spip/spip.php?article239\#nb5

Beguin Verbrugge A. \& Despres-Lonnet M. (2011) « Contexte matériel et humain des apprentissages »- in : A. Beguin-Verbrugge et S. Kovacs (éds.) Le cahier et l'écran (49-63). Paris : Hermes.

Bento M. (2016) ReVEA tâche 5 : Livrable 5.2 
Boubée N. (2007) Des pratiques documentaires ordinaires : analyse de l'activité de recherche d'information des élèves du secondaire. Toulouse 2.

Bouzon A., Couzinet V. \& Normand R. (1996) «Les doctorants livrés à la recherche documentaire »-Bulletin des Bibliothèques de France 41, 6 (54-59).

Chaudiron S. \& Ihadjadene M. (2010) « De la recherche de l'information aux pratiques informationnelles »-Études de Communication 35 (13-30).

Choppin A. (2008) «Le manuel scolaire, une fausse évidence historique »-Histoire de l'Éducation 117 (7-56)

Delamotte É., Liquète V. \& Frau-Meigs D. (2014) «La translittératie ou la convergence des cultures de l'information : supports, contextes et modalités »-Spirale 53 (145-156).

Diekema A. R. \& Olsen W. M. (2012) « The notion of relevance in teacher information behavior »-Proceedings of the American Society for Information Science and Technology 49, 1 (1-9).

Dioni, C. (2008) Métier d'élève, métier d'enseignant à l'ère numérique. https://edutice.archives-ouvertes.fr/edutice-00259563/

Gardiès C., Fabre I. \& Couzinet V. (2010) « Re-questionner les pratiques informationnelles »-Études de Communication 35 (121-132).

Gueudet, G. (2015). ReVEA tâche 3 : Livrable 3.1. http://www.cfem.asso.fr/actus-revea/livrables/livrable-revea-3.1

Gueudet G. \& Trouche L. (2010) Ressources vives : Le travail documentaire des professeurs en mathématiques. Rennes: PU Rennes.

Khaneboubi M. (2014) ReVEA tâche 2 : Livrable 2.1.

Levi Strauss C. (1965). La pensée sauvage. Paris : Plon.

Marcel J. F., Duperiez V., Périsset Bangnoud D., \& Tardif M. (2007) Coordonner, collaborer, coopérer. Louvain-la-Neuve : De Boeck.

Maurel D. \& Chebbi A. (2012) «La perception de la confiance informationnelle» - Communication et Organisation 42 (73-90).

Messaoui A. (2014) Influences des technologies numériques sur les pratiques professionnelles d'enseignants de collège; Mémoire de master, dir. C. Charnet ; Université Paul Valery, Montpellier.

https://www.academia.edu/15322042/Influences_des_technologies_num\%C3\%A9riqu es_sur_les_pratiques_professionnelles_denseignants_de_coll\%C3\%A8ge

Messaoui A. (2016) «Panorama du travail collectif au sein d'un collège rural »Présenté à ReVEA collectif, séminaire du 18 mars 2016, Lyon. http://ife.ens-lyon.fr/ife/recherche/groupes-de-travail/revea-collectif-mars2016/messaoui-mars-2016

Moiraud J.-P. (2009) «ENT et PLE opposition ou complémentarité ? » https ://moiraudjp.wordpress.com/2009/10/08/ent-et-ple-opposition-oucomplementarite

Passeron J. C. \& Revel J. (2005) Penser par cas. Paris : EHESS.

Pedauque, R. T. (2006) Le Document à la lumière du numérique. Caen : C\&F éditions.

Pepin B., Gueudet G. \& Trouche L. (2013) «Re-sourcing teachers' work and interactions : a collective perspective on resources, their use and transformation »-ZDM Mathematics education 45, 7 (929-943).

Perrault A. M. (2007) «An Exploratory study of biology Teachers' Online information seeking practices the collaborative relationship between school library media specialists and teachers » - School Library research 10. 
Perrenoud P. (1983) «La pratique pédagogique entre l'improvisation réglée et le bricolage »-Éducation \& Recherche 2 (198-212).

Pirolli, F. (2010) «Web 2.0 et pratiques documentaires »-Les Cahiers du Numérique 6,1 (81-95).

Rabardel P. (1995) Les hommes et les technologies : approche cognitive des instruments contemporains. Paris : Armand Colin.

Rocha K. de M. (2016) «Uses of online resources and documentational trajectories : the case of Sesamath »- Présenté à International Congress on Mathematical Education, Hambourg.

Sabra H. (2016) «L'étude des rapports entre documentations individuelle et collective : incidents, connaissances et ressources mathématiques »Recherches en Didactique des Mathématiques 36, 1.

Wang C. (2017) «Analyzing teachers' expertise, resources and collective work throughout Chinese and French windows »- Présenté à International Congress on Mathematical Education, Hambourg.

Wojciechowska A. (2011) «Pratiques documentaires de chercheurs à l'ère numérique : le cas des mathématiciens et informaticiens en France »Documentaliste - Sciences de l'Information 48, 3 (62). 


\section{ANNEXE : QUESTIONNAIRE}

\section{Ressources pour la classe}

1 [Ressources] Quels types de documents utilisez-vous pour faire la classe ? Choisissez la réponse appropriée pour chaque élément :

Toujours Souvent Rarement Jamais Sans réponse

$\begin{array}{lccccc}\text { Texte } & 11 & 17 & 2 & 0 & \\ \text { Audio } & 2 & 10 & 10 & 7 & 1 \\ \text { Vidéo } & 0 & 17 & 9 & 3 & 1 \\ \text { Image fixe } & 4 & 20 & 6 & 0 & 0 \\ \text { Autre } & 2 & 5 & 4 & 2 & 17\end{array}$

2 [Ressources] Si vous avez répondu « Autre », merci de préciser :

Veuillez écrire votre réponse ici :

Logiciels (7) Jeux (1) Objets techniques (1) Exposés d'élèves (1)

3 [Ressources] En règle générale, vous préférez créer entièrement vos supports de cours :

Veuillez sélectionner une seule des propositions suivantes ( 1 = pas du tout)

$\begin{array}{ll}1 & 0 \\ 2 & 2 \\ 3 & 6 \\ 4 & 13 \\ 5 & 9\end{array}$

4 [Ressources] En règle générale, vous préférez utiliser des supports de cours déjà tout prêts Veuillez sélectionner une seule des propositions suivantes ( 1 = pas du tout) :

$\begin{array}{ll}1 & 13 \\ 2 & 7 \\ 3 & 6 \\ 4 & 6 \\ 5 & 2 \\ & 0\end{array}$

5 [Ressources] Quelle(s) ressource(s) utilisez-vous pour préparer vos cours ? Choisissez la réponse appropriée pour chaque élément :

Le manuel de la classe

D'autres manuels papier

D'autres manuel en ligne

Des sites web liés à votre discipline

Des sites web généralistes

La presse spécialisée écrite (APMEP, NRP ...)

La presse spécialisée en ligne

Des logiciels généralistes (suite bureautique)

Toujours Souvent Rarement Jamais Sans réponse

Des logiciels spécialisés (Géogebra, Sketchup...

Autre :

\begin{tabular}{ccccc} 
Toujours & Souvent & Rarement & \multicolumn{4}{c}{ Jamais } & Sans réponse \\
5 & 8 & 11 & 4 & 2 \\
2 & 19 & 6 & 1 & 2 \\
0 & 11 & 5 & 10 & 4 \\
4 & 15 & 9 & 0 & 4 \\
2 & 13 & 9 & 2 & 4 \\
0 & 7 & 12 & 8 & 3 \\
0 & 8 & 12 & 8 & 2 \\
11 & 8 & 3 & 5 & 3 \\
2 & 9 & 4 & 11 & 4 \\
& 1 & & 2 & 27
\end{tabular}

6 [Ressources]Si vous avez répondu "Autre", merci de préciser :

Veuillez écrire votre réponse ici :

Des livres d'histoire de type universitaire 
7 [Recherche] Si vous utilisez des sites web liés à votre discipline, il s'agit de :

Choisissez toutes les réponses qui conviennent :

Site web disciplinaire académique $\quad 17$

Site web d'association professionnelle $\quad 12$

Blog ou site web personnel d'un enseignant 22

Edu'base nationale 3

Sans réponse

Autre : Big Challenge, BBC News (1), Educnet (1), tous les sites susceptibles d'appuyer les notions à

travailler (1), sites institutionnels (2)

8 [Manuel] Vous utilisez le manuel de classe :

Choisissez toutes les réponses qui conviennent :

Pour tout le travail de la classe 5

Pour introduire une nouvelle notion $\quad 7$

Pour faire des exercices avec les élèves en classe $\quad 14$

Pour donner des exercices aux élèves à la maison $\quad 11$

Vous n'utilisez pas le manuel de la classe 5

Sans réponse

Autre :

9 [Stockage] Où stockez-vous vos ressources pour la classe ?

Choisissez la réponse appropriée pour chaque élément :

\begin{tabular}{|c|c|c|c|c|}
\hline & $\begin{array}{c}\text { Stockage } \\
\text { permanent }\end{array}$ & $\begin{array}{l}\text { Stockage } \\
\text { temporaire }\end{array}$ & Jamais & Sans réponse \\
\hline Sur votre ordinateur personnel & 27 & 2 & 1 & 0 \\
\hline Sur votre ordinateur professionnel & 7 & 5 & 11 & 4 \\
\hline Sur l'intranet de l'établissement (ex : session Louise) & 2 & 3 & 17 & 8 \\
\hline Dans des classeurs / pochettes & 17 & 8 & 2 & 3 \\
\hline Dans des cahiers & 0 & 0 & 19 & 11 \\
\hline Sur une clé USB & 20 & 6 & 2 & 2 \\
\hline Sur un disque dur externe & 15 & 2 & 9 & 4 \\
\hline En ligne dans le cloud (Dropbox, ENT...) & 4 & 6 & 13 & 7 \\
\hline Autre : & & 1 & 2 & 27 \\
\hline
\end{tabular}

10 [Stockage] Si vous avez répondu « Autre », merci de préciser :

Veuillez écrire votre réponse ici : ENT (1) Je n'ai pas d'ordinateur professionnel (1)

11 [Recherche] Lorsqu'il vous manque un document ou une information pour préparer votre cours, vous :

Choisissez la réponse appropriée pour chaque élément :

Toujours Souvent Rarement Jamais Sans réponse

demandez à un(e) collègue

cherchez dans une revue professionnelle

cherchez sur un site web professionnel

$\begin{array}{ccccc}1 & 11 & 13 & 3 & 2 \\ 0 & 5 & 11 & 11 & 3 \\ 5 & 18 & 4 & 1 & 2 \\ 17 & 11 & 2 & 0 & 0 \\ 0 & 1 & 17 & 9 & 3 \\ 0 & 1 & 0 & 2 & 23\end{array}$

utilisez les ressources du CDI

Autre :

12 [Recherche] Si vous avez répondu "Autre", merci de préciser :

Veuillez écrire votre réponse ici : 
13 [Recherche]

Que pensez-vous de l'affirmation suivante : « Rechercher des ressources sur Internet vous fait gagner du temps »?

Veuillez sélectionner une seule des propositions suivantes :

Tout à fait d'accord 10

D'accord

10

Pas d'accord

9

Pas du tout d'accord

Sans réponse

14 [Recherche] Pourquoi?

Veuillez écrire votre réponse ici :

15 [Recherche] Lorsque vous incorporez des éléments trouvés sur Internet dans votre support de cours, avez-vous l'habitude d'indiquer leur origine?

Veuillez sélectionner une seule des propositions suivantes :

Oui 7

Non 22

16 [Collectif] Vous arrive-t-il de publier vos cours ou de partager vos ressources sur Internet (sur un blog, un site, sur Twitter...)

Veuillez sélectionner une seule des propositions suivantes :

Oui 4

Non 26

17 [Collectif] Si vous avez répondu oui, merci de préciser la fréquence et le(s) lieu(x) de publication Veuillez écrire votre réponse ici : ENT (3) ; site web académique (1)

18 [Préparation] Qu'est-ce qui a changé depuis les 3 dernières années dans votre manière de préparer vos cours?

Veuillez écrire votre réponse ici :

19 [Ressources] Quels seraient les critères d'une bonne ressource pour la classe ?

Veuillez écrire votre réponse ici :

20 [EMI]Est-ce que vous formez déjà vos élèves à l'éducation aux médias et à l'information (EMI) ? Veuillez sélectionner une seule des propositions suivantes :

Oui 7

Non

21 [EMI]Si oui, à quelle partie de votre programme avez-vous intégré l'EMI ?

Veuillez écrire votre réponse ici :

\section{Travail collectif dans ou hors l'établissement}

22 [Collectif] Quelles sont les thématiques de collaborations les plus fréquentes avec vos collègues, quelle que soit leur discipline?

Choisissez la réponse appropriée pour chaque élément :

Préparation de contrôle commun

Mise en place d'une progression

Préparation d'une séance

Toujours Souvent Rarement Jamais Sans réponse

Projet de classe ou de niveau

Système d'évaluation des élèves

Organisation pédagogique de l'équipe

Choix d'un nouveau manuel

$\begin{array}{ccccc}5 & 2 & 10 & 9 & 4 \\ 2 & 7 & 12 & 6 & 2 \\ 0 & 7 & 13 & 6 & 4 \\ 2 & 11 & 14 & 1 & 2 \\ 0 & 6 & 17 & 3 & 4 \\ 3 & 11 & 11 & 4 & 1 \\ 11 & 4 & 6 & 6 & 3\end{array}$


Toujours Souvent Rarement Jamais Sans réponse

\begin{tabular}{|c|c|c|c|c|}
\hline Réflexion sur un problème de votre discipline & 5 & 15 & 6 & 1 \\
\hline Réflexion sur une question de pédagogie & 2 & 18 & 6 & 1 \\
\hline Réflexion sur une question de la profession & 4 & 18 & 4 & 1 \\
\hline Autre & 1 & 1 & 0 & \\
\hline
\end{tabular}

23 [Collectif] Si vous avez répondu « Autre », merci de préciser

Veuillez écrire votre réponse ici : pas de collègue dans la discipline (1) Conseil sur un logiciel (1)

24 [Collectif]Depuis le début de l'année scolaire, vous avez collaboré avec au moins 1 collègue de VOTRE DISCIPLINE :

Veuillez sélectionner une seule des propositions suivantes :

$\begin{array}{ll}\text { Systématiquement } & 5 \\ \text { Souvent } & 7 \\ \text { De temps en temps } & 9 \\ \text { Rarement } & 3 \\ \text { Jamais } & 3 \\ \text { Sans réponse } & 3\end{array}$

25 [Collectif] Depuis le début de l'année scolaire, vous avez collaboré avec au moins 1 collègue d'une AUTRE DISCIPLINE :

Veuillez sélectionner une seule des propositions suivantes :

$\begin{array}{ll}\text { Systématiquement } & 4 \\ \text { Souvent } & 9 \\ \text { De temps en temps } & 5 \\ \text { Rarement } & 6 \\ \text { Jamais } & 3 \\ \text { Sans réponse } & 3\end{array}$

26 [Collectif] Quel est votre rôle dans le cadre de ces collaborations ?

Choisissez toutes les réponses qui conviennent :

Initiateur du projet $\quad 18$

Contributeur 15

Animateur 9

Rôle administratif 4

Sans réponse 7

Autre :

27 [Collectif]Vous échangez ou préparez des séances pédagogiques avec un professeur :

Choisissez la réponse appropriée pour chaque élément :

du collège ET de votre discipline

du collège ET d'une autre discipline

Toujours Souvent Rarement Jamais Sans réponse

d'un autre établissement ET de votre discipline

$\begin{array}{lllll}4 & 5 & 16 & 4 & 1\end{array}$

d'un autre établissement ET d'une autre discipline

$\begin{array}{lllll}0 & 6 & 12 & 10 & 2 \\ 3 & 6 & 8\end{array}$

$\begin{array}{lllll}3 & 6 & 8 & 12 & 1\end{array}$

28 [Collectif] Lorsque vous collaborez avec des collègues, quels outils utilisez-vous ? Choisissez toutes les réponses qui conviennent :

$\begin{array}{ll}\text { le téléphone } & 14 \\ \text { la messagerie électronique } & 29 \\ \text { une liste de diffusion } & 1 \\ \text { un forum de discussion } & 0 \\ \text { un réseau social } & 0 \\ \text { une dropbox } & 3\end{array}$


$\begin{array}{ll}\text { Sans réponse } & 0 \\ \text { Autre : } & 5 \\ \text { Contact direct } & (4)\end{array}$

29 [Collectif] Quels ont été les motifs professionnels pour vous engager dans de telles collaborations ? Choisissez toutes les réponses qui conviennent :

Se former

5

Échanger des expériences 23

Échanger des ressources 18

Se plier à des obligations professionnelles 7

Sans réponse

Autre :

Formation

Vos projets pour la rentrée 2016

30 [cycle] Sur quels niveaux souhaiteriez-vous enseigner à la rentrée 2016 ? (QCM) Choisissez toutes les réponses qui conviennent :

$\begin{array}{ll}6 \mathrm{e} & 16 \\ 5 \mathrm{e} & 21 \\ 4 \mathrm{e} & 19 \\ 3 \mathrm{e} & 22 \\ \text { ULIS } & 1\end{array}$

31 [EPI] Dans quelle(s) thématique(s) des EPI envisagez-vous de vous investir ? Choisissez toutes les réponses qui conviennent :

Monde économique et professionnel

Culture et création artistiques 16

Langues et cultures de l'Antiquité

Information, communication, citoyenneté 7

Corps, santé, bien-être et sécurité

Transition écologique et développement durable 5

Sciences, technologie et société

Langues et cultures étrangères/régionales.

Je ne souhaite pas m'investir dans un EPI

6
10
11
7
7
5
6
5
2

32 [EPI] Sur quel(s) niveau(x) pensez-vous mettre en œuvre un EPI ?

Choisissez toutes les réponses qui conviennent :

$\begin{array}{ll}5 \mathrm{e} & 18 \\ 4 \mathrm{e} & 8 \\ 3 \mathrm{e} & 9 \\ \text { Sans réponse } & 4\end{array}$

33 [EPI] Avec quelle(s) discipline(s) pensez-vous collaborer dans les EPI ?

Choisissez toutes les réponses qui conviennent

Français 13

Anglais 7

Espagnol 4

Allemand 4

Éducation musicale 3

Documentation 7

EPS 7

Mathématiques 5

Histoire géographie EMC $\quad 8$

SVT 


\section{A. MESSAOUI}

Sciences physiques $\quad 4$

Arts plastiques $\quad 11$

Lettres classiques $\quad 8$

Technologie 6

Aucune

34 [programme] Pour la mise en œuvre des nouveaux programmes, vous avez commencé à :

Choisissez toutes les réponses qui conviennent :

Lire les textes parus au BO

Lire les commentaires sur les nouveaux programmes dans la presse professionnelle 9

Lire les commentaires sur les nouveaux programmes dans la presse syndicale

Préparer des ressources de cours

Participer à des formations

Former des collègues

Discuter avec vos collègues

Participer à un groupe d'étude ou de recherche 3

Aucune démarche entamée pour le moment 4

Autre :

35 [programme] Ressentez-vous un manque de ressources pour mettre en œuvre les nouveaux programmes ?

Veuillez sélectionner une seule des propositions suivantes :

Oui

17

Non

36 [Ressources]Si oui, de quelles ressources auriez-vous besoin ?

Veuillez écrire votre réponse ici :

Manuels - exemple de mise en œuvre (7)

Programme par niveau (5)

\section{Histoire professionnelle}

37 [Étude] Pouvez-vous décrire votre parcours universitaire ?

Discipline Spécialité Année

$B a c$

DEUG

Licence

Maîtrise

$D E A$

Master

Doctorat

CAPES

Agrégation

38 [Étude] En quelle année avez-vous commencé à enseigner ?

Veuillez écrire votre réponse ici :

39 [Carrière] Depuis combien d'années enseignez-vous dans votre établissement actuel ?

Veuillez écrire votre réponse ici :

40 [Carrière] Êtes-vous TZR cette année ?

Veuillez sélectionner une seule des propositions suivantes :

Oui 
41 [Carrière] Êtes-vous contractuel ?

Veuillez sélectionner une seule des propositions suivantes :

Oui

Non

42 [Carrière] Quels sont les 3 derniers établissements où vous avez exercé avant d'arriver dans cet établissement?

$$
\begin{gathered}
\text { Établissement d'exercice } \\
\text { (collège ou lycée) }
\end{gathered} \text { Académie } \begin{gathered}
\text { Nombre d'années Particularité } \\
\text { en poste }
\end{gathered}
$$

2

3

43 [Carrière] Exercez-vous ou avez-vous exercé des responsabilités particulières au sein ou en dehors de l'établissement ?

Choisissez toutes les réponses qui conviennent :

$\begin{array}{ll}\text { Tuteur de stagiaire } & 12\end{array}$

Formateur

Professeur principal 24

Coordinateur de discipline $\quad 12$

Participation active dans une association professionnelle 1

Participation à un cercle d'étude $\quad 5$

Je n'ai pas exercé de responsabilité particulière $\quad 2$

Sans réponse

Autre: $\quad 4$

44 [Carrière] Avez-vous eu dans votre parcours, d'autres expériences professionnelles ?

Veuillez sélectionner une seule des propositions suivantes :

Oui

Non

45 [Carrière] Si vous avez répondu oui, merci de préciser lesquelles :

Veuillez écrire votre réponse ici :

46 [ID] Quel est votre âge ?

Veuillez écrire votre réponse ici :

47 [ID] Quelle discipline enseignez-vous ?*

Veuillez sélectionner une seule des propositions suivantes :

$\begin{array}{lr}\text { Allemand } & 1 \\ \text { Anglais } & 5 \\ \text { Arts plastiques } & 1 \\ \text { Documentation } & 0 \\ \text { Éducation musicale } & 1 \\ \text { EPS } & 1 \\ \text { Espagnol } & 2 \\ \text { Français } & 4 \\ \text { Histoire géographie EMC } & 4 \\ \text { Lettres classiques } & 1 \\ \text { Mathématiques } & 5 \\ \text { Sciences physiques } & 1 \\ \text { SVT } & 2 \\ \text { Technologie } & 1\end{array}$

48 [ID] Si vous acceptez d'être contacté pour réaliser un entretien, merci d'indiquer votre nom et votre prénom. 
Répondre à cette question seulement si les conditions suivantes sont réunies :

La réponse était «Anglais » ou «Mathématiques » à la question « 47 [ID] » (Quelle discipline enseignez-vous ? ) et La réponse était « Anglais « ou « Mathématiques » à la question « 47 [ID] » (Quelle discipline enseignez-vous?)

Veuillez écrire votre réponse ici :

Merci d'avoir participé à cette enquête ! 